\title{
Localized eosinophilic gastroenteritis with necrotizing granulomas presenting as acute abdomen
}

\author{
G. Fraile, J.L. Rodríguez-García, R. Beni-Pérez ${ }^{1}$ and C. Redondo ${ }^{2}$ \\ Departments of Internal Medicine, 'Surgery and ${ }^{2}$ Pathology, Hospital Ramón y Cajal, Spain
}

\begin{abstract}
Summary: Eosinophilic gastroenteritis is a condition of unknown aetiology that has been frequently reported involving the stomach and bowel. The colon is rarely cited as a site for the condition. We report a patient suffering from allergic symptoms (asthma, atopic dermatitis, elevated serum IgE levels) who presented with colonic perforation due to eosinophilic colitis with necrotizing granulomas.
\end{abstract}

\section{Introduction}

Idiopathic eosinophilic infiltration of the gut has been classified into diffuse and localized forms as clinical and pathological related disorders. ${ }^{1}$ Various sites, from the oesophagus to the rectum, may be involved and present with symptoms and signs ranging from abdominal discomfort to acute intestinal obstruction. Histological evidence of a predominantly eosinophilic infiltration of gastrointestinal mucosa, in the absence of parasitic infection or vasculitis, confirms the diagnosis of eosinophilic gastroenteritis. ${ }^{2.3}$

We report the case of a patient with localized eosinophilic colitis leading to perforation of the colon. The clinical course of the patient and the occurrence of necrotizing granulomas on biopsy are discussed.

\section{Case report}

A 38 year old man was admitted because of abdominal pain and fever. A history of asthma and atopic eczema was recorded. On physical examination temperature was $37.5^{\circ} \mathrm{C}$. He complained of pain localized in the right lower quadrant with rebound tenderness. Laboratory data revealed that the haematocrit was $43 \%$, the white cell count was $11 \times 10^{9} / 1$ without eosinophilia or neutrophilia. The blood urea, nitrogen, glucose, bilirubin, calcium, total protein, electrolytes and amylase were normal. A clinical diagnosis of acute appendicitis was made and the patient underwent surgery. The appendix was normal but there was a mass, $4 \mathrm{~cm}$ in diameter, at the right colon with perforation of its

Correspondence: J.L. Rodríguez-García, M.D., $\mathrm{Dr}$ Collado Piña 2, 1-D, 02003 Albacete, Spain.

Accepted: 12 November 1993 wall. An exudative fluid was seen around the caecum. A right hemicolectomy with resection of $11 \mathrm{~cm}$ of terminal ileum was performed.

Examination of the resected specimen showed a colonic mass located near the ileocaecal valve. Pathological study showed a massive inflammatory infiltrate affecting the entire colonic wall, although it was more prominent in the submucosa, pericolic fatty tissue and peritoneal surface. The inflammatory infiltrate consisted of sheets of eosinophils and necrotizing epithelioid granulomas with giant cells (Figure 1). Necrosis was of the 'eosinophilic' type, with free eosinophils, granules and Charcot-Leyden crystals (Figure 2). Similar changes were found on three lymph nodes studied. The search for Mycobacterium tuberculosis, parasites and fungi was negative, and there was no evidence of vasculitis. Pathological examination of the appendix was normal.

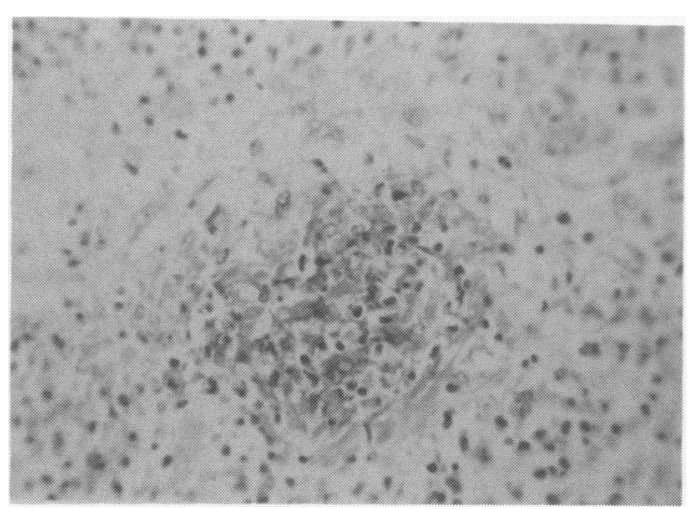

Figure 1 Inflammatory infiltrate composed almost exclusively of eosinophils. A well-formed granuloma is seen in the centre of the field with a multi-nucleated cell and 'threads' of eosinophilic necrosis. 


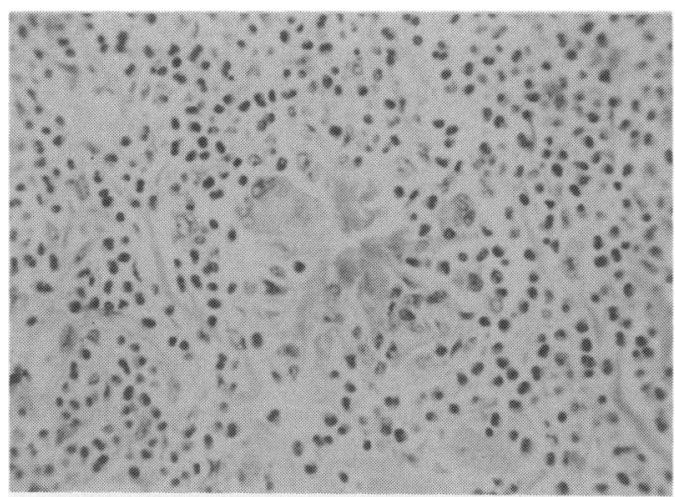

Figure 2 In this picture a large focus of eosinophilic necrosis is shown. Eosinophils and cellular debris are intermingled with the necrotic material which resembles 'flames.'

Further studies included haematological and biochemical profiles that were normal. The total eosinophil count was $500 \times 10^{9} / 1$. Sputum and urine culture were negative for $M$. tuberculosis. Antibodies to aspergillus antigens were not demonstrable in serum. Examination of the stool for ova and parasites was negative. Elevated serum IgE levels were found. Results of skin tests and RAST to multiple foods and inhalants were negative. Upper gastrointestinal endoscopy and barium study of the small intestine disclosed no abnormalities.

The patient recovered well after surgery. He was treated with prednisolone for several weeks followed by maintenance therapy with ketotifen. One year later, the patient remains free of symptoms.

\section{Discussion}

Localized eosinophilic gastroenteritis is a circumscribed form of eosinophilic infiltration of the gut not associated with peripheral eosinophilia or atopic history. It is most frequently located near the pylorus, although it may be found throughout the gastrointestinal tract. The colon is rarely cited as a site of the condition: in a series of 40 patients, eosinophilic infiltration of the colon was documented in only two cases. ${ }^{1}$ Patients may present with signs and symptoms of pyloric outlet obstruction, appendicitis or an acute abdomen from intestinal involvement. This form does not respond to corticosteroid therapy, and may require excision because of pyloric obstruction or other symptoms. Recurrence has not been reported after surgical excision. ${ }^{4}$

Diffuse eosinophilic gastroenteritis most commonly involves the stomach and the proximal small intestine with eosinophilic inflammation of either the mucosa, submucosa or serosa. It has a chronic course with recurrent disease characterized by abdominal pain, nausea, vomiting and diarrhoea. Other reported clinical manifestations include weight loss, anaemia, melaena, protein-losing enteropathy, oesophagitis and intestinal perforation. Many patients have findings suggestive of allergic disorders (allergic rhinitis, atopic dermatitis, asthma, elevated IgE levels). ${ }^{5,6}$ Steroids are the mainstay of therapy for eosinophilic gastroenteritis. ${ }^{7}$ Ketotifen may represent an effective alternative to corticosteroids, ${ }^{8}$ while the value of oral cromoglycate remains controversial. ${ }^{9}$ Moreover, a spontaneous benign course in patients with acute abdomen due to eosinophilic colitis has been reported. ${ }^{10}$

Opinions are divided about whether diffuse and localized eosinophilic gastroenteritis are variants of the same disease. ${ }^{1}$ The case here reported is in agreement with the concept that these two forms are related conditions, since the patient presented with localized eosinophilic infiltration of the colon, but protean-associated allergic manifestations (asthma, atopic dermatitis and elevated IgE concentrations in serum) were recorded.

A remarkable feature, rarely recorded in cases of eosinophilic gastroenteritis, ${ }^{11}$ was the presence of necrotizing granulomas, similar to the allergic granulomas described by Churg and Strauss. ${ }^{12}$ It is suggested that the limited form of eosinophilic infiltration of the gut is at the benign end of the spectrum of the hypereosinophilic syndrome, and that the disease of allergic granulomatous angiitis (Churg-Strauss syndrome) is at the other end. This concept of a disease continuum is supported by cases showing progression from the limited form to the disseminated form or to allergic granulomatosis. ${ }^{13}$ Moreover, vasculitis of small vessels has been also noted on biopsy specimens from patients with eosinophilic gastroenteritis. ${ }^{11}$ It must be noted that no vasculitic involvement could be seen in our case and that, at the present time, the patient has not developed clinical data of allergic granulomatosis (hypereosinophilia, nervous system disease, renal disease or skin manifestations) other than asthma.

Laparotomy is often required to provide the diagnosis of eosinophilic colitis: 14 patients, from a series of 20 cases of eosinophilic colitis, underwent surgery to exclude carcinoma, presumed appendicitis or for unremitting symptoms despite medical treatment. ${ }^{6}$ Clinical presentation with intestinal perforation emphasizes that this unusual condition must be included in the differential diagnosis of an acute abdomen, particularly in those patients with previous allergic symptoms, even in the absence of peripheral eosinophilia. Eosinophilic gastroenteritis with serosal involve- 
ment can be the cause of peritonitis in atopic patients and conservative measures may be indicated. ${ }^{14}$ However, we believe that surgery for diagnostic and therapeutic purposes cannot be

\section{References}

1. Talley, N.J., Shorter, R.G., Philips, S.F. \& Zinsmeister, A.R. Eosinophilic gastroenteritis: a clinicopathological study of patients with disease of the mucosa, muscle layer and subserosal tissues. Gut 1990, 31: 54-58.

2. Johnstone, J.M. \& Morson, B.C. Eosinophilic gastroenteritis. Histopathology 1978, 2: 335-348.

3. Zora, J.A., O'Connell, E.J., Sachs, S.I. \& Hoffman, A.D. Eosinophilic gastroenteritis: a case report and review of the literature. Ann Allergy 1984, 53: 45-47.

4. Heyman, M.B. Food sensitivity and eosinophilic gastroenteropathies. In: Sleisinger and Fordtran (eds). Gastrointestinal Disease, 4th edn. W.B. Saunders Company, Philadelphia, 1989, pp. 1113-1134.

5. Moore, D., Lichtman, S., Lentz, J. et al. Eosinophilic gastroenteritis presenting in an adolescent with isolated colonic involvement. Gut 1986, 27: 1219-1222.

6. Naylor, A.R. \& Pollet, J.E. Eosinophilic colitis. Dis Col Rect 1985, 28: 615-618.

7. Case records of the Massachusetts General Hospital (case 20-1992). N Engl J Med 1992, 326: 1342-1349.

8. Melamed, I., Feany, S.J., Sherman, P.M. \& Roifman, Ch.M. Benefit of ketotifen in patients with eosinophilic gastroenteritis. Am J Med 1991, 90: 310-314. delayed, since in those patients perforation of the colonic wall may be the first manifestation of a localized eosinophilic gastroenteritis.

9. Heatley, R.V., Harris, A. \& Atkinson, M. Treatment of a patient with clinical features of both eosinophilic gastroenteritis and polyarteritis nodosa with oral chromoglycate. Dig Dis Sci 1980, 25, 470-472.

10. Barbera, L. \& Zumtobel, V. Acute abdomen in eosinophilic colitis. Leber Magen Darm 1990, 20, 293-295.

11. Suen, K.C. \& Burton, J.D. The spectrum of infiltration of the gastrointestinal tract and its relationship to other disorders of angiitis and granulomatosis. Hum Pathol 1979, 10: 31-43.

12. Shimamoto, Ch., Hirata, I., Ohshiba, S. et al. Churg-Strauss syndrome (allergic granulomatous angiitis) with peculiar multiple colonic ulcers. Am J Gastroenterol 1990, 85: 316-319.

13. Manko, M.A., Cooper, J.H. \& Myers, R.N. Disseminated hypereosinophilic disease. Am J Gastroenterol 1972, 57: 318-325.

14. Case records of the Massachusetts General Hospital (case 30-1993). N Engl J Med 1993, 329: 343-349. 